\title{
Field Applications with CAPSY
}

\section{Leon DeVos, TNO-BOUW, PO.box 49, 2600 AA Delft, The Netherlands. Bob Hasara, Spectra Physics Laserplane, 5475Kellenburger Rd. Dayton, $O H$.}

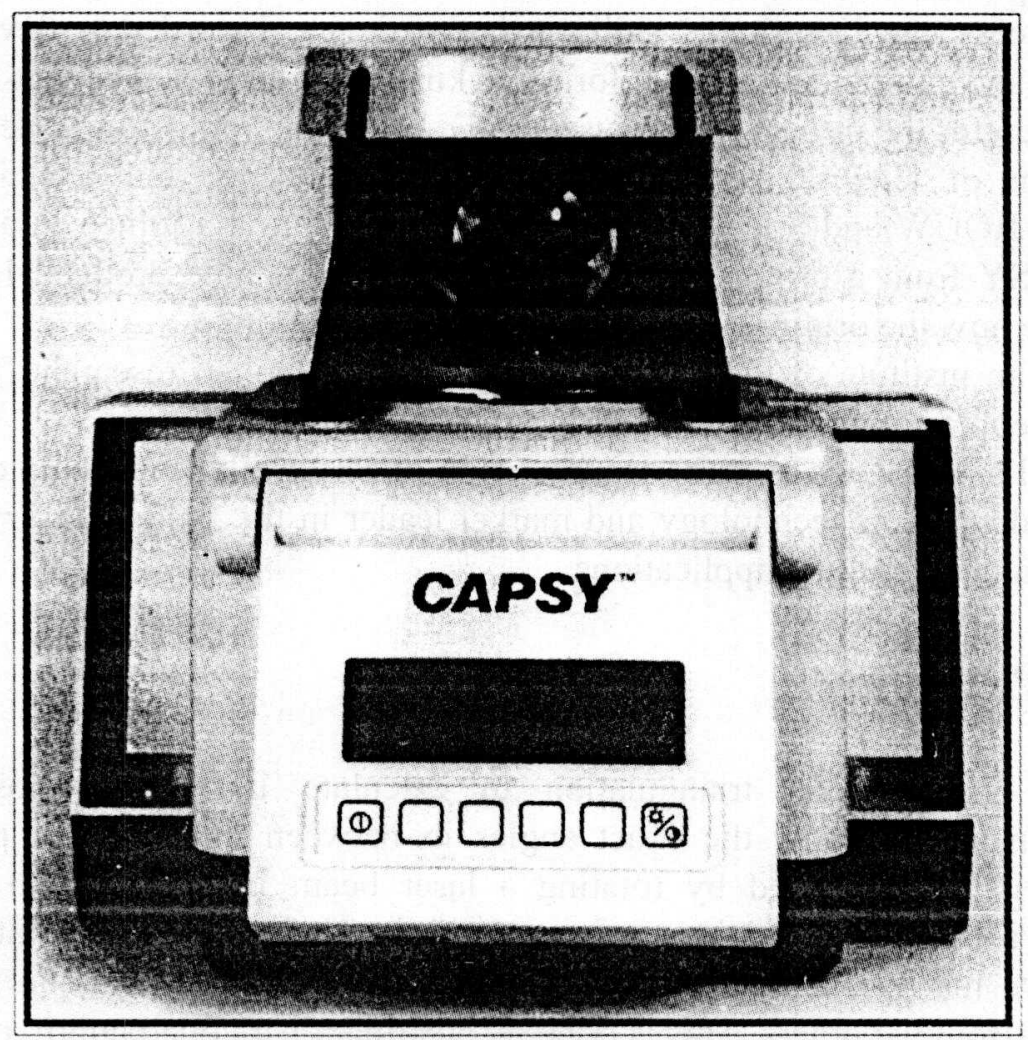

\begin{abstract}
In June 1989 CAPSY has been introduced for the first time at the ISARC' 89 in San Francisco. CAPSY (Computer Aided Positioning SYstem) is intended to be a lowcost real-time location tool for construction. Operating in working area's with dimensions like 300 by 500 feet it is capable of locating itself to an accuracy of $+/-1 / 8$ of an inch in $\mathrm{X}$ - and Y-direction. Since this introduction CAPSY positioning system has grown from a lab-prototype to a commercially available product now. The initial specifications are met( low cost, accurate, portable) and in some cases even exceeded. Although CAPSY positioning system can be used as just a sophisticated measuring and locating tool there might be an even more important role to fulfil in the future. Because of the onboard computer capabilities CAPSY can be the link between the design stage of the building process (CAD-systems, highly computerised) and the actual building process at the job site(no computerisation at all). Transferring data from the design to CAPSY makes it possible to use CAPSY as a one-man survey instrument. At the same time it can function as a real world digitizer on the job site to feed back the 'as build' situation .
\end{abstract}




\section{INTRODUCTION}

In 1989 a project was started at TNO-BOUW to investigate how unhealthy and hazardous working conditions on the construction site could be avoided. The general feeling was that automation and robotization were part of the solution. Not to an extreme situation of eliminating all human labour on the job site ( very unrealistic especially in construction ) but trying to let the construction worker be the brains and the (automated) equipment to be the muscles. In that case the worker is always in control and the building process can benefit his skills and experience. In order to develop more automated tools and equipment the need for some kind of location device was recognised immediately. Therefor the project was focused in the beginning on getting such a device. After the design of CAPSY and verifying several concepts and techniques with prototypes TNO-BOUW and Spectra Physics Laserplane started a joint venture in order to develop CAPSY from a prototype to a commercially available product. Having this product available now the original goals of the project can continue.

TNO-BOUW is an institute of the Dutch organisation for applied research TNO. There are about 6000 people employed in this non-profit organisation.

Spectra Physics Laserplane is an American division of Spectra Physics, employing 5400 people world wide. SPL is technology and market leader in the field of laser equipment for construction and agriculture applications.

\subsection{How CAPSY works.}

CAPSY is based on triangulation to calculate its current position. For triangulation it needs to know the exact angles in between three known points as a reference. This is accomplished by rotating a laser beam in a horizontal plane and scanning the environment for the three reference points. These reference points are made of retro-reflective material and each so called 'reflector' is made unique in order to distinguish them from the others. This is done by creating a bar code pattern of the retroreflective material. (In section 1.2 is explained how these reflectors become known points.) When the laser beam is hitting the reflector the laser light is reflected back into the unit and analysed by the internal computer. This will provide two essential pieces of information. First of all it will measure the exact angle of this reflector in respect to an internal index and secondly it will recognise the specific bar code of this reflector so it knows which target is scanned. The angle is measured with an accuracy of 0.001-0.003 of a degree. This is done by an internal computer controlled angular measuring system which is virtually insensitive to rotational velocity fluctuations. For construction applications the rotational speed will be $300 \mathrm{rpm}$. Angular errors due to being off level are compensated in software rather then by a mechanical compensator. An in built XY-tilt sensor measures the amount of degrees off level in both axis. The total portable unit is 
approximately 6 by 6 by 8 inches and weights about $6 \mathrm{lb}$. Rechargeable battery packs last about 4 hours.

\subsection{Getting started with CAPSY.}

Before CAPSY can be used as a one-man survey instrument there needs to be done some initial work. The user has to prepare the working area and the unit for operation. This needs to be done only. once for every working area and the information will remain in the unit until it is deleted by the user itself. The most important part of initialising a working area is the so called 'set up' procedure. This procedure calculates the still unknown XY-position of the reflectors. As part of preparing the working area the user has placed 3 or more reflectors at the periphery of the area. The triangulation algorithm requires the XY-position of the reference points. It would be very inefficient if it was necessary to survey the positions of the reflectors by a survey crew. Because CAPSY is able to calculate very accurate angles it is capable also to calculate the XYposition of the reflectors. Assuming there are always at least two known reference points marked on the floor by a surveyor, CAPSY uses these points and their co-ordinates as a base for calculating the position of the reflectors. After entering these co-ordinates into the unit the user has to place CAPSY on reference point \#1. CAPSY will take about 30 seconds to sample and store the angles of the recognised reflectors. The next step is to place CAPSY on the second reference point. Again CAPSY will sample and store for about 30 seconds the angles of the recognised reflectors. After this CAPSY is able to calculate the XY-positions of the reflectors. The complete set-up procedure is guided to the user by CAPSY itself and takes about 3 to 5 minutes. As long as the reflectors stay in the working area the unit is ready for operation and no new set ups are necessary.

\subsection{CAPSY Advisor.}

As mentioned before the user has to place at least three reflectors around the working area to make CAPSY work. Reflectors are recognised by CAPSY at 150 feet maximum under a plus or minus 45 degree acceptance angle (horizontal plane). Typically a user will place more than just three reflectors for several reasons. More reflectors means a larger working area. As soon as CAPSY recognise three reflectors it will produce a XYposition. So it is very easy to enlarge the working area by placing more reflectors at certain distances along the periphery of the area. A second reason is to create a full coverage for a irregular shaped working area. Another reason is that sometimes a reflector is blocked. In that case a three reflector system can't calculate a position. CAPSY scans 5 times a second for reflectors. If it recognise more than 3 reflectors it means it has more than one 3 reflector combinations, resulting in more than one solution for this particular position. In that case an evaluation algorithm becomes active. This algorithm will select the best combination of reflectors for this position. Choosing the best reflector combination is very important to get the desired accuracy of $+/-1 / 8$ of an inch. In irregular shaped areas it can be difficult for an inexperienced user to determine where to 
place the reflectors. A software tool called CAPSY Advisor might be of great help for such a user. After entering the shape of the area CAPSY Advisor will suggest locations for reflectors and present a map of the expected accuracy.

\subsection{CAPSY's field book.}

CAPSY is used for setting out points and for measuring points. This implies that there is a lot of data involved in the form of XY-coordinates and identifiers. CAD systems or specific survey software may generate lists of points to be down loaded in CAPSY. Digitised points needs to be up loaded to a computer system. All this data handling is supported through the field book. The field book is a pocket calculator kind of device and communication is performed through a RS232 serial link. It is capable to link to CAPSY and every other computer/device having a RS232 interface. The software in the field book takes care of the data transport between the two devices. On top of that it functions as an extended display and keyboard for CAPSY and as an temporary memory storage for lists of points.

\section{GENERAL CONSTRUCTION APPLICATIONS}

\subsection{XY-positioning.}

Calculating a new XY-position is the most basic function of CAPSY. All other functions are derived from this function. The actual position is updated 5 times a second and can be considered there for as a real time position. The simplest way to do XYpositioning is to use the XY-coordinate mode which displays on the LCD screen the current position. By comparing this actual position on the LCD screen with the desired position one should be able to find the right spot on e.g. the floor and mark it. Although this looks easy in practice it is difficult to get any sense for distance and direction based on a comparison of two co-ordinates. More helpful to the user will be when the (list of) co-ordinates are down loaded into the unit. In that case CAPSY is able to guide the user to the desired spot. This is possible because CAPSY not only knows the current position but it calculates it's bearing in respect to the reflectors too. The current position, the desired position and the current bearing is all the information it needs to guide the user to the next position. The way how this is displayed to user can vary. It can be expressed in a distance forward/backward combined with a distance left/right. That is a numerical method. A more intuitive way to present it is some kind of graphical display. CAPSY's LCD screen is a character display ( graphic LCD's don't perform well at lower temperatures) but a limited graphic display is made possible. By using a non linear scale and an iconised presentation of CAPSY and the desired position it is possible to position the unit within 1/8 of inch on the desired spot guided by this pseudo graphic display. This can be done without interpreting any numerical information. Basically the position CAPSY calculates is a position of a point inside CAPSY. This internal point is inaccessible and for that reason worthless because it can not be used to mark or point to a 

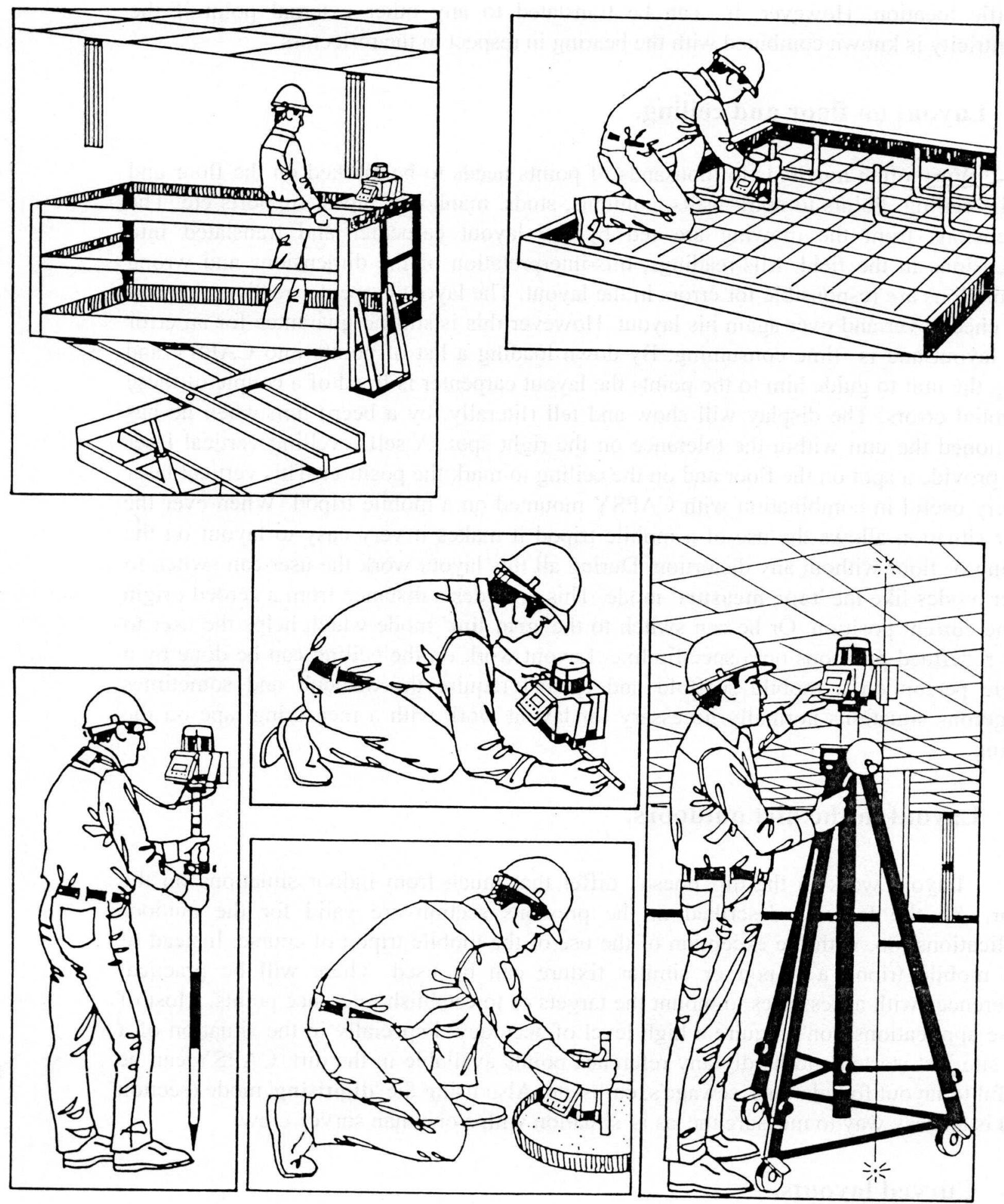

Some field situations with CAPSY 
specific location. However, it can be translated to any other external point if the eccentricity is known combined with the bearing in respect to the reflectors.

\subsection{Layout on floor and ceiling.}

Very often hundreds to thousands of points needs to be marked on the floor and on the ceiling. Points to mark walls, columns, studs, manholes, pipes, supports etc. The dimensions from the drawing are red by the layout carpenter and translated into dimensions in the field. Mis-readings, mis-interpretation of the dimensions and wrong calculations are responsible for errors in the layout. The layout carpenter realises this and will check over and over again his layout. However this is still no guarantee for an error free layout and is time consuming. By down loading a list of points into CAPSY and using the unit to guide him to the points the layout carpenter is freed of a couple of these potential errors. The display will show and tell (literally, by a beep) him when he has positioned the unit within the tolerance on the right spot. A self levelling vertical laser will provide a spot on the floor and on the ceiling to mark the position. This vertical laser is very useful in combination with CAPSY mounted on a mobile tripod. When ever the floor situation allows the use of a mobile tripod it makes it very easy to layout on the ceiling or floor without any distortion. During all this layout work the user can switch to other modes like the 'tape measure' mode. This provides a distance from a zeroed origin to the current position. Or he can switch to the 'grid line' mode which helps the user to find redefined positions on a specific line. Layout work on the ceiling can be done by a single person on a mobile scaffold and doesn't require the difficult and sometimes dangerous situations normally necessary for layout work with a measuring tape on the ceiling.

\subsection{Layout in the dirt outdoors.}

Layout work in the dirt doesn't differ that much from indoor situations on the floor. All the features described in the previous section are valid for the outdoor applications too, with the exception of the use of the mobile tripod of course. Instead of this mobile tripod a bi-pod or similar fixture can be used. There will be practical differences with accessories to mount the targets or to establish reference points. Most of these applications don't require a high level of accuracy. Especially in the situation of a job site just started and hardly any reference points available in the dirt, CAPSY can be useful to layout foundations, sewage's, curbs etc. Also using the 'digitising' mode (section 2.5) is an easy way to measure the 'as is' situation with a one man survey crew.

\subsection{Curved layouts.}

The fact that CAPSY calculates 5 times a second it's actual position is a gateway to a whole range of possibilities. One of them is the so called 'curved' mode. This mode 
gives the user the opportunity to layout very irregular shaped lines without the need of the numerous necessary co-ordinates. Simply by entering the shape of the curved line in terms of tangent points and radii into the field book, it will be pre-processed and ready for down loading into CAPSY. When this is done and the 'curved' mode is active CAPSY will indicate every time it intersects this curve. The user is able to position the unit very accurate on the curve and to make a mark. So the number of marks is not limited and the user can make as many marks as he thinks what is necessary for a proper layout.

\subsection{Digitising the real world.}

CAPSY can be used as a real world digitiser too. Indoors and outdoors. Digitising an existing situation is very often required to produce up to date drawings as part of the preparations for a construction. Even during the construction process very often already existing situations are digitised in order to verify and monitor the building process. The 'digitise' mode allows the user to identify and measure more than 2000 points by a one man survey crew. Later on these points can be up loaded into the field book and/or PC for further processing.

\section{SPECIAL APPLICATIONS}

\subsection{Machine Control.}

So far CAPSY is discussed as an one man, portable, real time positioning instrument. Because CAPSY is highly computerised and because it provides a real time position it may be used as a positioning sensor for machine control applications. Especially the fact that CAPSY is aware of it's bearing within the co-ordinate system makes it applicable to mount on e.g. earth moving machines, pile foundation machines and AGV's. There are two grades of integrating CAPSY in such a systems. When they are operated by a man, it may provide the information about position and bearing on a screen. It is the operators responsibility how to use this information. The other way is to integrate CAPSY in the control loop of the system and than there will be no human interference.(Overhead cranes,AGV's, Tunnel Drilling Robot). It is very likely that those applications require a special version of CAPSY.(physical and software).

\subsection{Industrial.}

Basically CAPSY is a real time, accurate, angle measuring device. It is the embedded software which transforms it into a positioning instrument. Although normally the angle measuring part is not visible to the user, it is available by relatively simple software modifications. By mounting reflective tape on specific construction parts or machine parts it can monitor very slight movements over long periods without any human interference. Highly accurate alignments over long distances is another application. It is the users imagination which limits the applications. 


\section{CONCLUSION}

It took 4 years to develop CAPSY from a concept proved by a crude prototype to a real product, ready for mass production as it is now. Still you may consider the product is only just born. There is yet a lot to learn. A difficulty is that this product has no history. There is practically nothing known about how this kind of technology will be accepted on the construction site. Total stations and electronic theodolites are of course no novelty any more, but these are operated by a highly educated category of people. Very important will be the user interface and the easiness of use. Unlike it's technical performance (which can be tested and improved without user input) the user interface needs to be shaped and refined by feedback from the users in the field. It will be of extreme importance to listen to their comments and critics. Fortunately most of the modifications as result of this user feed back will be software modifications. Anticipating to go through several iterations the computer hardware and the embedded sotftware of CAPSY are prepared to handle these software updates very easily. The first field tests ( started Jan. 1993) are indicating an excellent technical performance of the instrument. As expected the user interface went through a it's first iteration and is ready for a next sequence of field tests. These uncertainties about the user interface are of course only related to the 'one man positioning crew' version of CAPSY. A machine control or industrial application will communicate via the standard RS232 interface conform a special protocol. The over all conclusion is that there is definitely a place for CAPSY on the construction site and there may be even more (industrial) applications which can use an accurate, real time, low cost positioning sensor.

\section{REFERENCES}

1. [DeVos '89] The Computer Aided Positioning SYstem CAPSY, A low cost positioning system for construction. Leon DeVos, Hans Schouten, TNO-BOUW, The Netherlands. 6 Th. ISARC, June 1989, San Francisco, USA.

2. [Krom '92] Automation of Robot Operation Planning Based on Product Modelling.

Ronald Krom, Frits Tolman, TNO-BOUW, The Netherlands. 9 Th. ISARC, June 1992, Tokyo, Japan.

3. [Krom '93] The development of a drilling robot for the installation of railway tracks.

Ronald Krom, Ruud Kloek, TNO-BOUW. The Netherlands. prof. Charles Vos. Delta Marine Consultants. The Netherlands. 10 Th. ISARC, June 1993, Houston, USA. 